\title{
Barrier height distributions - the influence of weak channels
}

\author{
A. Trzcińska1,a, W. Czarnacki ${ }^{2}$, P. Decowski ${ }^{3}$, M. Kisieliński $^{1,2}$, P. Koczoń ${ }^{4}$, A. Kordyasz ${ }^{1}$, E. Koshchiy ${ }^{5}$, \\ M. Kowalczyk ${ }^{1,6}$, B. Lommel ${ }^{4}$, E. Piasecki ${ }^{1,2}$, K. Rusek ${ }^{1,2}$, I. Strojek ${ }^{2}$, A. Stolarz ${ }^{1}$, and K. Zerva ${ }^{7}$ \\ 1 Heavy Ion Laboratory, University of Warsaw, Warsaw, Poland \\ 2 The Andrzej Sołtan Institute for Nuclear Studies, Świerk/Otwock, Poland \\ 3 Smith College, Northampton \\ 4 GSI Helmholtzzentrum fur Schwerionenforschung GmbH, Darmstadt, Germany \\ 5 Kharkiv University, Kharkiv, Ukraine \\ 6 Institute of Experimental Physics, University of Warsaw, Warsaw, Poland \\ 7 University of Ioannina, Ioannina, Greece
}

\begin{abstract}
The barrier distributions for the ${ }^{20} \mathrm{Ne}+{ }^{58,60,61} \mathrm{Ni}$ systems were experimentally determined. In the ${ }^{58} \mathrm{Ni}$ case one observes a distinct "structure" in the distribution (two peaks) which is partially damped for ${ }^{60} \mathrm{Ni}$ and completely smoothed out for ${ }^{61} \mathrm{Ni}$. It seems that smoothing is caused by non-collective excitations of the target nuclei.
\end{abstract}

It has been demonstrated experimentally in many systems that the fusion barrier between two nuclei does not have a unique value but rather a weighted distribution of heights, $D_{\text {fus }}$. Measurements of barrier distributions are a long-term project of our group.

$D_{\text {fus }}$ can be determined directly from fusion excitation function measurements [1]. There is also an alternative method: it has been shown both theoretically and experimentally that the rather difficult fusion measurements can be replaced by much simpler quasi-elastic scattering measurements at backwards angles, giving rise to the barrier height distribution $D_{q e}$. The basic equivalence of these two methods is confirmed by many existing data sets [2, 3].

Studies of fusion cross-section at sub-barrier energies show that there is a relation between the reaction mechanism and the structure of the interacting nuclei reflected in the shape of the barrier height distribution. The best theoretical description of this observable can be made within the Coupled-Channels (CC) method where the interplay between various reaction channels is taken into account. In some cases in a distribution one observes a significant structure being a fingerprint of the couplings involved [4, 5].

In our experiments we have focused on the ${ }^{20} \mathrm{Ne}$ projectile, since this nucleus has extremely large deformation parameters: $\beta_{2}=0.46, \beta_{3}=0.39, \beta_{4}=0.27$ [6-8]. We have performed a series of barrier distribution measurements for several targets: ${ }^{112,116,118} \mathrm{Sn},{ }^{n a t} \mathrm{Ni},{ }^{90,92} \mathrm{Zr}$ and ${ }^{208} \mathrm{~Pb}$.

The results of the first measurements were surprising: the barrier distributions for ${ }^{20} \mathrm{Ne}+{ }^{112,116,118} \mathrm{Sn}$ turned out to

\footnotetext{
a e-mail: agniecha@slcj.uw.edu.pl
}

be smooth [9] in contradiction to theoretical predictions, while for the ${ }^{20} \mathrm{Ne}+{ }^{\text {nat }} \mathrm{Ni}$ system a clearly structured distribution [10] was obtained, in very good agreement with the $\mathrm{CC}$ calculations taking into account collective excitations of the reaction partners.

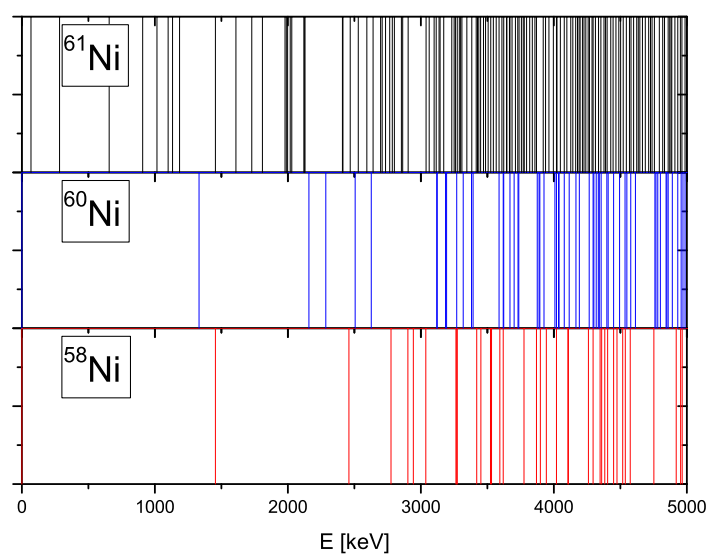

Fig. 1. Adopted level densities in ${ }^{58,60,61} \mathrm{Ni}$ (from www.nndc.bnl.gov/nudat2).

In a series of experiments [11] with $\mathrm{Zr}$ isotopes we have proved that excitation of the non-collective states can give rise to a significant smoothing of the structure of the barrier height distribution $D_{q e}$. The theoretical investigation of the influence of non-collective excitations on reaction dynamics in low-energy reactions, and in particular on quantum tunneling, may be found in $[12,13]$. Follow- 

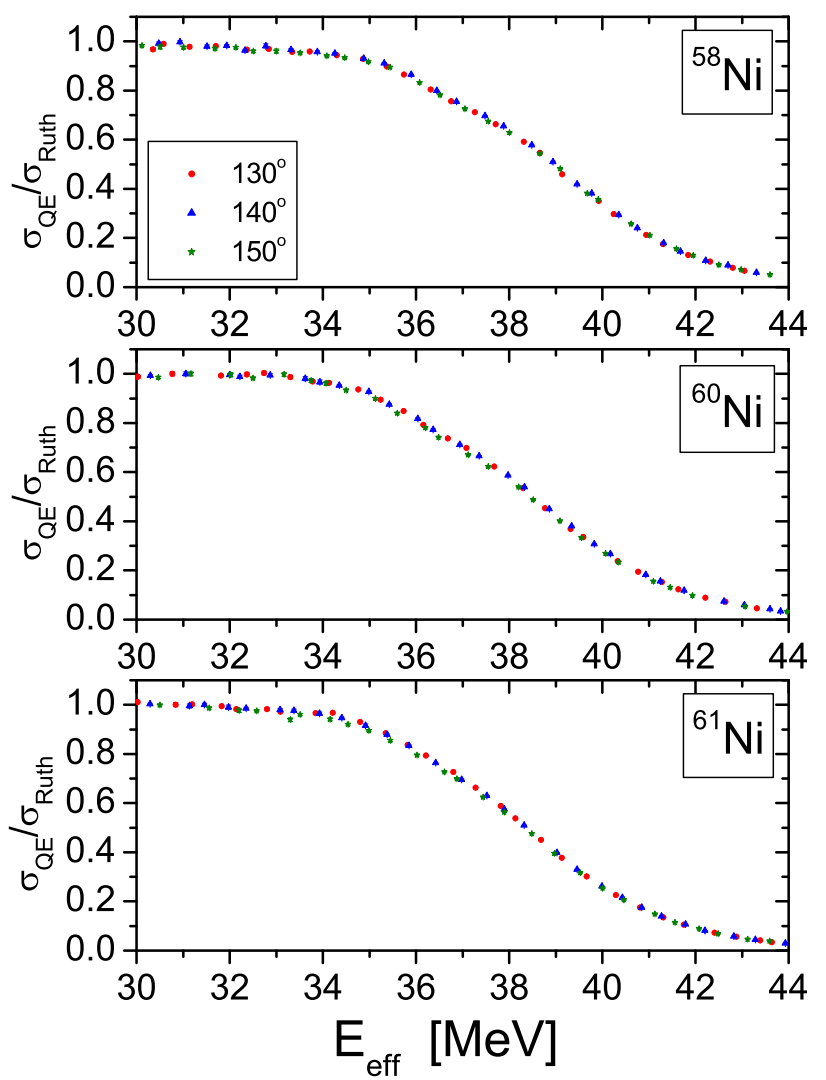

Fig. 2. Excitation function for quasi-elastic backscattering of ${ }^{20} \mathrm{Ne}$ on ${ }^{58,60,61} \mathrm{Ni}$ targets registered at $130^{\circ}, 140^{\circ}$ and $150^{\circ}$ (in the lab.).

ing the conclusions from our ${ }^{20} \mathrm{Ne}+{ }^{90,92} \mathrm{Zr}$ experiments we expected that the higher the level density of the target nucleus the stronger should be the smoothing of the distribution. To check this hypothesis we performed measurements with three $\mathrm{Ni}$ isotopes $\left({ }^{58,60,61} \mathrm{Ni}\right)$ which have very different level densities (Fig. 1) and determined their barrier height distributions. The experiment was performed at the Heavy Ion Laboratory, University of Warsaw. The experimental technique was as described in [14]. The results are presented in Fig. 2: excitation functions and in Fig. 3: barrier height distributions. The "effective energy", $E_{\text {eff }}$ is defined as in [2]: $E_{\text {eff }}=\frac{2 E}{1+\operatorname{cosec}(\theta / 2)}$ (where $E$ is the beam energy and $\theta$ is the scattering angle in c.m.s.). The results are in agreement with our expectations: for the semi-magic ${ }^{58} \mathrm{Ni}$ where the level density is low the barrier distribution is structured, for ${ }^{60} \mathrm{Ni}$ the structure is less marked whereas for ${ }^{61} \mathrm{Ni}$, where the level density is the highest, the structure at $37 \mathrm{MeV}$ is completely smoothed out (Figs. 3 and 4). The energy resolution for all cases was the same: FWHM $\simeq 0.8 \mathrm{MeV}$. In Fig. 4 the barrier distribution for all isotopes are compared. As the barrier height is the proportional to $1 / R \sim 1 / A^{1 / 3}$, the $E_{e f f}$ for ${ }^{60} \mathrm{Ni}$ and ${ }^{61} \mathrm{Ni}$ were re-normalized by the factor $(A / 58)^{1 / 3}$.

One should emphasize that according to CC calculations, taking into account collective effects only, the shape of all three distributions should be virtually the same.

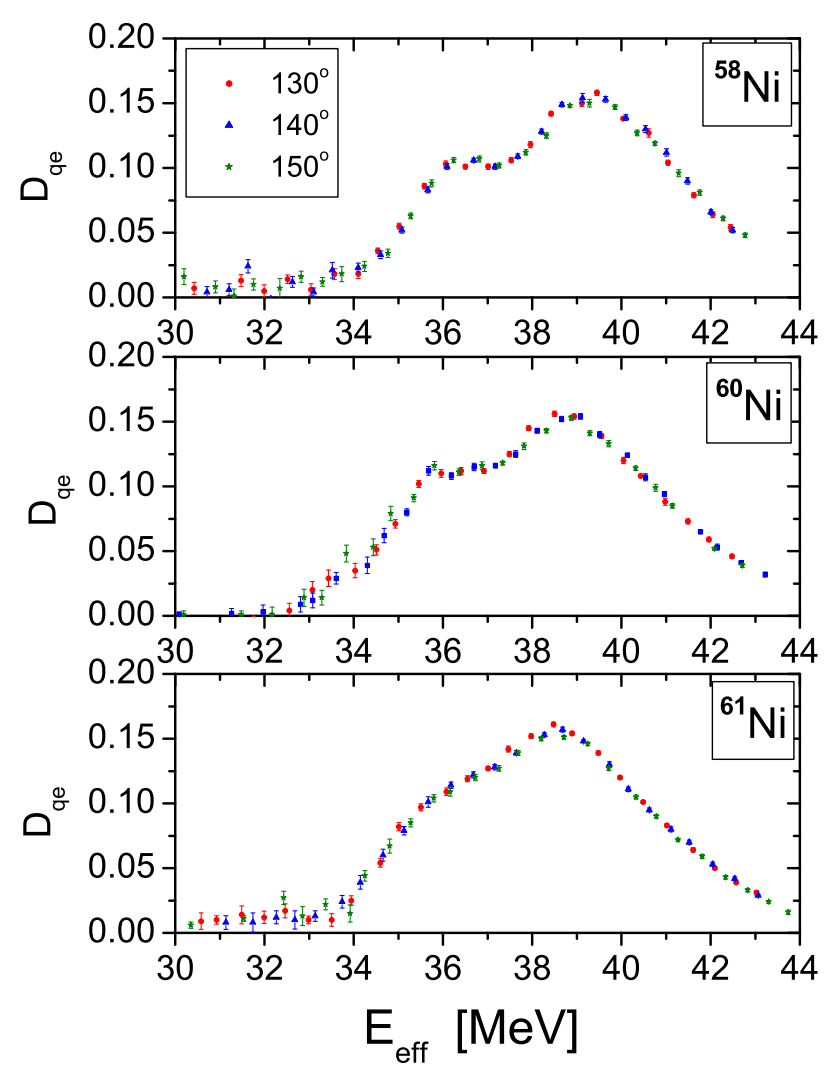

Fig. 3. Barrier height distributions for the ${ }^{20} \mathrm{Ne}+{ }^{58,60,61} \mathrm{Ni}$ systems derived from the excitation functions presented in Fig. 2 using the relation $D_{q e}=-d\left(\frac{\sigma_{q e}}{\sigma_{\text {Ruth }}}\right) / d E$.

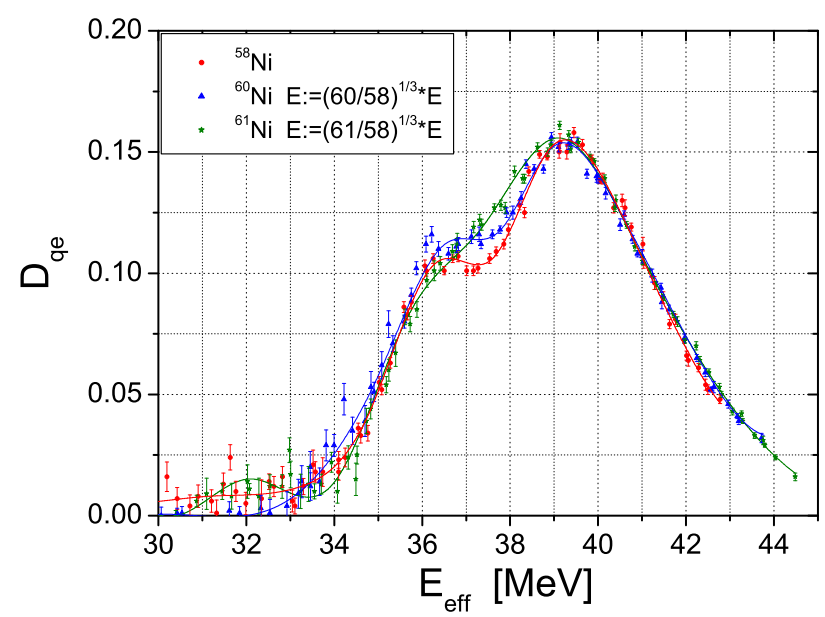

Fig. 4. Comparison of barrier height distributions shown in Fig. 3 after renormalization of of barrier heights for ${ }^{60} \mathrm{Ni}$ and ${ }^{61} \mathrm{Ni}$ taking into account in an approximate way sizes of the target nuclei (see the explanation in the text). A spline approximation of distributions are marked with lines to guide an eye. 


\section{FUSION11}

The influence of the transfer channels on the barrier distribution structure for the same systems was the subject of an experiment performed very recently. The evaluation of the data is still in progress.

\section{References}

1. N. Rowley et al., Phys. Lett. B 254, 25 (1991).

2. H. Timmers et al., Nucl. Phys. A 584, 190 (1995).

3. L. F. Canto et al., Phys. Repts. 424, 1 (2006).

4. M. Dasgupta et al., Annu. Rev. Nucl. Part. Sci. 48, 401 (1998).

5. A. M. Stefanini et al., Phys. Rev. Lett. 75, 864 (1995).

6. S. Raman et.al., At. Data and Nucl. Data Tabl. 78, 1 (2001).

7. R. H. Spear, At. Data and Nucl. Data Tabl. 42, 55 (1998).

8. G. Blanpied et al., Phys. Rev. C38, 2180 (1988).

9. L. Świderski et al., Int. Journ. of Modern Phys. E 13, 315 (2004).

10. L. Świderski et al., Int. Journ. of Modern Phys. E 14, 341 (2005).

11. E. Piasecki et al., Phys. Rev. C 80, 054613 (2009).

12. S. Yusa et al., Phys. Rev. C 82, 024606 (2010).

13. A. Diaz-Torres et al., Phys. Rev. C 82, 054617 (2010).

14. E. Piasecki et al., Phys. Rev. C 65, 054611 (2002). 